\title{
Paul Klee et les formes musicales: analogies enrichissantes
}

\author{
Paul Klee e as formas musicais: \\ analogias enriquecedoras
}

\author{
Zélia Chueke \\ Universidade Federal do Paraná / IReMus \\ zchuekepiano@ufpr.br
}

Resumé: Dans cet article, le dialogue entre les arts, notamment entre la musique et les arts plastiques, viendra enrichir notre relation avec les œuvres ci explorées. Les contours la division des plans sonores et plastiques, le groupement des motifs, l'idée du tout et des parties seront ainsi dévoilés dans le propos de mettre en valeur l'expérience interartistique.

Mot-clefs: Paul Klee; formes musicales; relations interartistiques.

Resumo: Neste artigo, o diálogo entre as artes, notadamente entre a música e as artes visuais é proposto enquanto fator de enriquecimento de nossa relação com as obras aqui exploradas. Os contornos e planos melódicos sonoros e plásticos, o agrupamento de motivos bem como a ideia do todo e das partes serão assim desvendados com o propósito de valorizar a experiência interartística.

Palavras-chave: Paul Klee; formas musicais; relações interartísticas.

\section{1 - Art et communication}

L'objectif de ce texte n'est pas l'élaboration d'une théorie au sujet de la relation entre les arts, mais l'enrichissement de cette relation, en promouvant également les particularités de chacune des différentes formes de manifestation 
artistique $^{1}$. Le dialogue entre les arts, naturellement libéré de toutes barrières, existe depuis le début du parcours de l'expression artistique qui est logiquement rattaché à celui de l'histoire même de l'humanité.

Depuis les premiers temps, la fonction primordiale de toutes les manifestations artistiques est sans aucun doute la communication. Les découvertes archéologiques en France et en Espagne montrent que les activités de chasse bien réussies sont enregistrées en détail à travers les peintures sur les murs des cavernes - manifestation d'art visuel - pendant les cérémonies de célébration qui incluaient vraisemblablement aussi bien le chant que la danse. Sont ici présents le son, le mouvement et l'image.

Dans ce texte nous traiterons justement de la relation entre le son et l'image, et le «mouvement » du discours dans chaque domaine. Dans tous les cas, le constat de cette relation ne fera pas la soustraction de la valeur individuelle de chaque forme d'expression artistique et ne fera surtout pas la substitution d'une forme d'art par une autre. Disons plutôt qu'elle viendra enrichir notre relation avec l'oeuvre sans jamais occuper la place de l'effet causé par l'oeuvre elle-même. Dans le domaine de la literature, Lessing (1990, p. 50-51) affirme par exemple $\mathrm{qu}^{\prime}$ " il n'est pas possible de traduire dans d'autres langages la peinture musicale des mots du poète ».

\section{2 - Musique et arts plastiques}

Prenons quelques exemples des relations entre la musique et l'image à partir de l'initiative de plusieurs peintres. Dans Musique du crépuscule V (1966Figure 1), Joan Miró (1893-1983) nous suggère les figures musicales, mais c'est le poète Pierre Reverdy (Erben 1998, p.174-175) qui nous éclaire à ce propos:

" L'image est une création pure de l'esprit. Elle ne peut naître d'une comparaison, mais du rapprochement de deux réalités plus ou moins éloignées. Plus les rapports des deux réalités rapprochées seront lointains et justes, plus l'image sera forte plus elle aura de puissance émotive et de réalité poétique. »

\footnotetext{
${ }^{1}$ Je tiens à remercier Jean-Yves Bosseur pour la relecture attentive de mon article et pour la contribution qu'il en a apporté compte tenu de sa spécialité dans le domaine des dialogues et de l'interaction entre la musique et les arts plastiques en tant que compositeur et chercheur. Cet article est représentatif d'une conférence présentée le 20 février 2009 à la Maison de la Recherche, Université Paris-Sorbonne, dans le cadre des Séminaires Musiques et Arts Plastiques sous la responsabilité de Mme. la Professeur Michèle Barbe.
} 


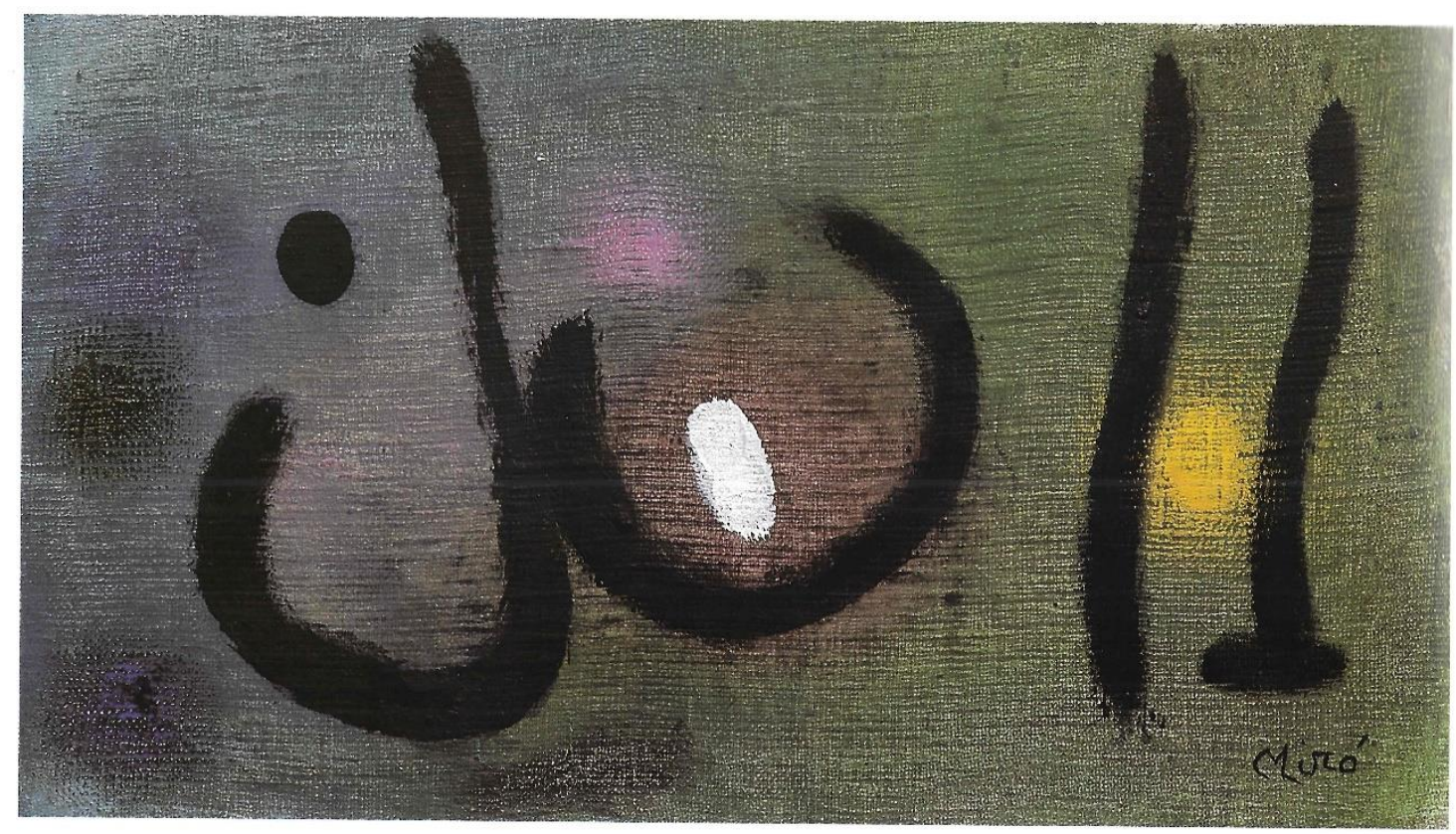

Figure 1: Jean Miró, Musique du crépuscule $V$

Un autre exemple est l'œuvre de Fernand Khnopff (1858-1921) intitulée En écoutant du Schumann (1883) ${ }^{2}$. La scène nous propose une ambiance typiquement bourgeoise de la fin du 19e siècle où l'on voit une dame très distinguée assise. La musique est suggérée par l'apparition partielle au fond de la salle d'un piano droit joué par quelqu'un qui touche les notes les plus aiguës. La décoration discrète - si l'on considère l'époque et la condition sociale suggérée - privilégie la musique qui remplit l'espace. Le modèle est la mère du peintre, décédée la même année, et Schumann était son compositeur préféré jusqu'à plus tard, quand le peintre connaîtra la musique de Wagner.

Dans les exemples mentionnés ci-dessus, l'expérience est essentiellement et primordialement visuelle, pouvant évidemment être enrichie à partir des données musicales fournis par les artistes eux-mêmes, par exemple à partir des titres des œuvres, ensuite à travers plusieurs détails visuels. Néanmoins, pour que les suggestions musicales transforment vraiment le processus de communication, le récepteur devrait être pourvu d'un certain niveau de formation musicale ainsi qu'avoir, pour ainsi dire, le matériau sonore gardé dans sa mémoire auditive.

Pour donner un autre exemple de la même relation, suggérée cette fois-ci par des musiciens, nous avons choisi Claude Debussy, qui affirmait aimer «presque autant les images que la musique »(Goubault 2002, p.12-15) ${ }^{3}$. Nous pouvons mentionner plusieurs de ses œuvres évoquant à la fois image et mouvement (soit à travers le discours musical et le scénario suggéré, soit à travers

\footnotetext{
2 http://users.skynet.be/litterature/symbolisme/kschumann.htm

${ }^{3}$ L'auteur explore le « vocabulaire des arts plastiques » utilisé par Debussy.
} 
les gestes $\mathrm{du}$ peintre et $\mathrm{du}$ musicien dans leur travail). Examinons les deux Arabesques, œuvres basées dans les deux positions du ballet classique également représentées par Degas dans les sculptures en bronze de sa série de danseuses - les trois cahiers des Images, les Estampes, qui établissent ainsi les connections entre l'expérience visuelle et auditive. Le graphisme des partitions des œuvres pour piano de Debussy est un exemple puissant des relations immédiates entre le visuel et le sonore; il " parle à l'œil autant qu'à l'oreille » (Goubault 2002, p.10). Les contours mélodiques, l'agroupement des motifs, la division des plans sonores et même le doigté sont naturellement implicites dans l'enregistrement graphique du matériau sonore. Nous soulignons ici la même idée que précédemment: les allusions, les descriptions, les analogies explorées par les musicologues, ne pourraient jamais faire en sorte que l'écoute intérieure du récepteur donne naissance à un contour mélodique ou découvre une ligne de cohérence, quelque soit le matériau sonore, si celui-ci ne disposait pas tout d'abord d'une richissime mémoire auditive et d'une certaine formation musicale.

\section{2 - Fugue, pastorale et polyphonie selon Paul Klee}

Le rôle évidemment important et solide représenté par la musique dans diverses œuvres de Paul Klee (1879-1940) est sans doute le résultat de son parcours musical. Fils de musiciens (Hall 1992, p.5) ${ }^{4}$, marié avec une pianiste (Ibid.) $)^{5}$ et, de plus, ayant reçu lui-même une formation musicale, la musique fait partie presque constamment de son expression artistique.

Pour prolonger nos constats antérieurs et instaurer une transition avec ce cas particulier, prenons le texte de Pierre Boulez (2008, p.4) autour de Paul Klee Le compositeur commente la forte impression provoquée chez Klee par Pelléas et Mélisande de Debussy, suivie du contact avec les tableaux de Cézanne, soulignant le fait que la comparaison Debussy-Cézanne n'a pas eu lieu et que Klee évitait les correspondances directes. "On dirait qu'il se méfie des transpositions littérales » (Boulez 2008, p. 44).

Selon Boulez, Klee sentait intuitivement les relations entre peinture et musique plutôt « dans une arborescence de translations invisibles qui se tissent à la racine même de la pensée créatrice » (Ibid.). Jugeant évidente la relation entre l'œil et l'oreille établie par le peintre, il mentionne sa perception de la " géométrie graphique et vibratoire de Bach » aussi bien que sa compréhension de «la structure des phrases, leurs relations à la forme, la logique du développement » (Boulez 2008, p. 46). Il rend évident le fait que « cette polyphonie, présente en permanence dans son esprit », faisait partie de son regard: “C'est évident, l'œil

\footnotetext{
${ }^{4}$ Son père était professeur de musique et sa mère avait étudié la musique.

${ }^{5}$ Lily Stumpf. Les soirées musicales faisaient partie de sa vie en famille.
} 
de Klee écoute. [...] Et il ose parfois la transcription graphique du contrepoint [...] ». Evidement tout cela est la conséquence de sa formation musicale antérieure, certainement fort solide.

\section{1 - La fugue de Klee et celle de Bach}

L'influence et la source d'inspiration dans notre premier exemple est la fugue (Hall 1992, p.60) dans Fuge in rot (1921), dont le thème présenté à plusieurs reprises ne fait qu'accentuer la forme, et nous pouvons observer ce fait dans les diverses sections musicales, l'exposition, les épisodes successifs, le passage du Stretto, ainsi qu'à travers les figures superposées dans le tableau en question.

Carducci-Augustin (1926, p. 482) suggère une idée philosophique du thème, conçu comme un germe, un noyau, une synthèse, un principe générateur de son propre développement. Cette idée est parfaitement en accord avec la description mentionnée ci-dessus et s'applique également, par exemple, à la Fugue no XVI du Clavier Bien Tempéré de Johann Sebastian Bach.

Dans cette fugue de Bach, le thème s'impose à travers une particularité : occupant seulement une mesure et demie, sa présence évidente dans le contresujet, les motifs qu'il génère sont présents dès le début du morceau. Cette relation intense entre le thème (Exemple 1a), le contre-sujet (Exemple 1b) et les motifs qui se déroulent autour d'eux mêmes (Exemples 1c e 1d), tirés de la terminaison du sujet, tout cela varié au début du contre-sujet pendant les trois épisodes ${ }^{6}$, conduit notre écoute à travers la trame du discours musical de la même façon que la relation entre thème et motifs nous conduisent visuellement à travers l'œuvre de Klee (Exemple 2). Evidement, ce parcours visuel peut être aussi auditif mais cela est directement lié à la formation musicale de l'observateur.
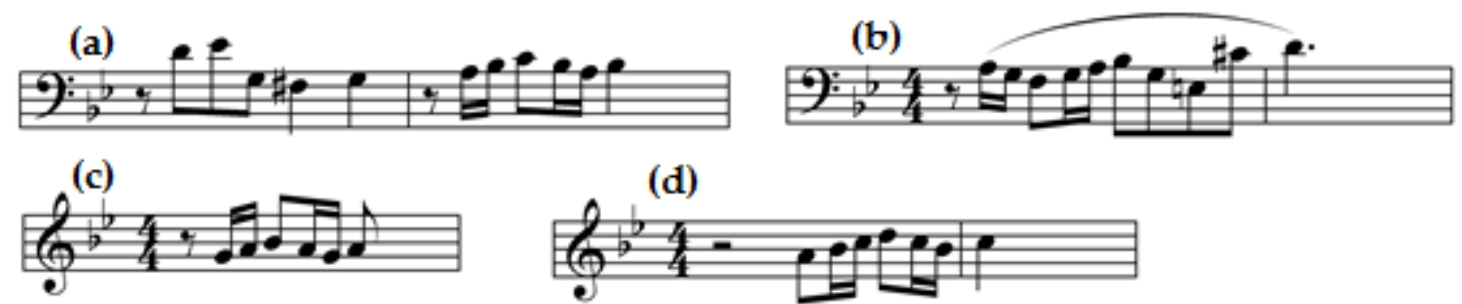

(d)

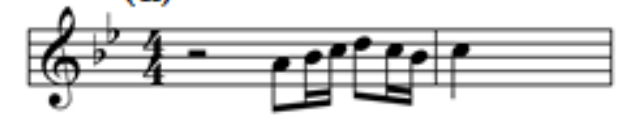

Exemple 1: (a) sujet, mes. 1-2.3; (b) contre-sujet, mes. 3-4.1;

(c) motif 1 , mes. 4.1.2-3.1; (d) : motif 2 , mes. 4.3.2-5.1

${ }_{6}$ Ep.1, mes.8-18.3; Ep.2, mes.18.3.2-24.3.1; Ep.3, mes.24.3.2-28.1 et le Stretto (mes.28.2-34). Plusieurs interprétations formelles sont possibles, comme un 4 ème épisode au lieu du Stretto suggéré par Homero Magalhães (1988, p. 165). 
Chez Klee, nous voyons le thème dans la figure qui s'impose au centre du tableau, suggérant une " résonance ». Il se répète, légèrement modifié, toujours accompagné d'une autre forme, plus géométrique, qui peut être comparée au contre-sujet. Les motifs réitérés de cette figure sont distribués parmi les autres apparitions du thème et son contre-sujet, produisant une sensation d'unité (Figure 2). La suggestion de résonance est appliquée à toutes les figures, renforçant également l'unité de l'image. Toutes les formes révèlent une forte liaison avec le thème, aspect évident, de même que l'énonciation du thème dans une fugue est évidente même pour des oreilles profanes. Il s'impose sur la toile et c'est la forme qui reste dans la mémoire du récepteur. Sa relation avec les autres formes s'intensifie vers le bas du tableau, comme le Stretto à la fin de la fugue.
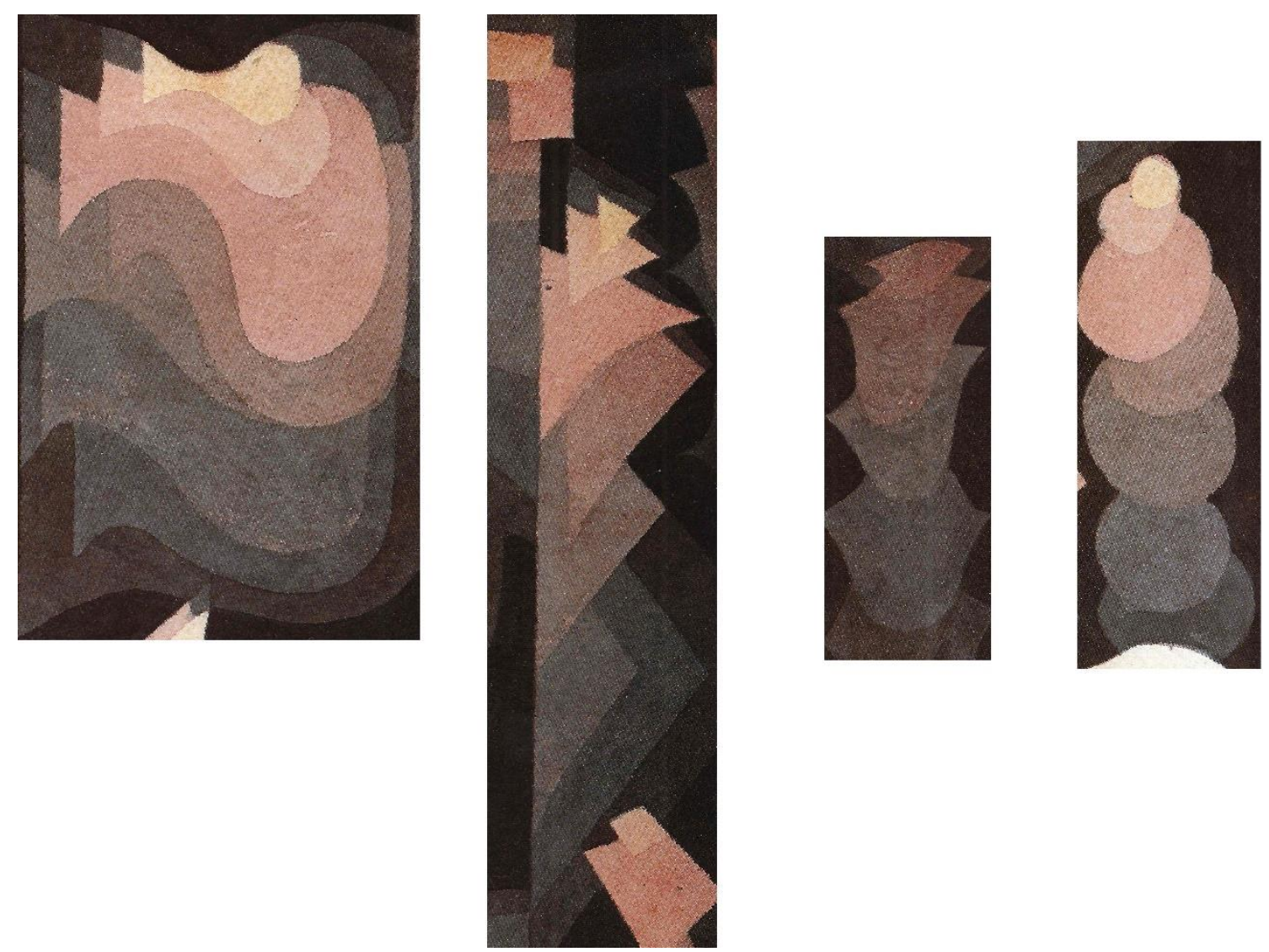

Figure 2: Suggestion de thème, contre-sujet, motif 1 e motif 2 dans Fuge in rot (1921) de Paul Klee

Dans la fugue, la visualisation du thème guide le musicien à travers le discours musical; l'imagination musicale, inscrite dans sa mémoire auditive, organise le matériau sonore en termes de hiérarchie, construisant une interprétation ou une analyse. 

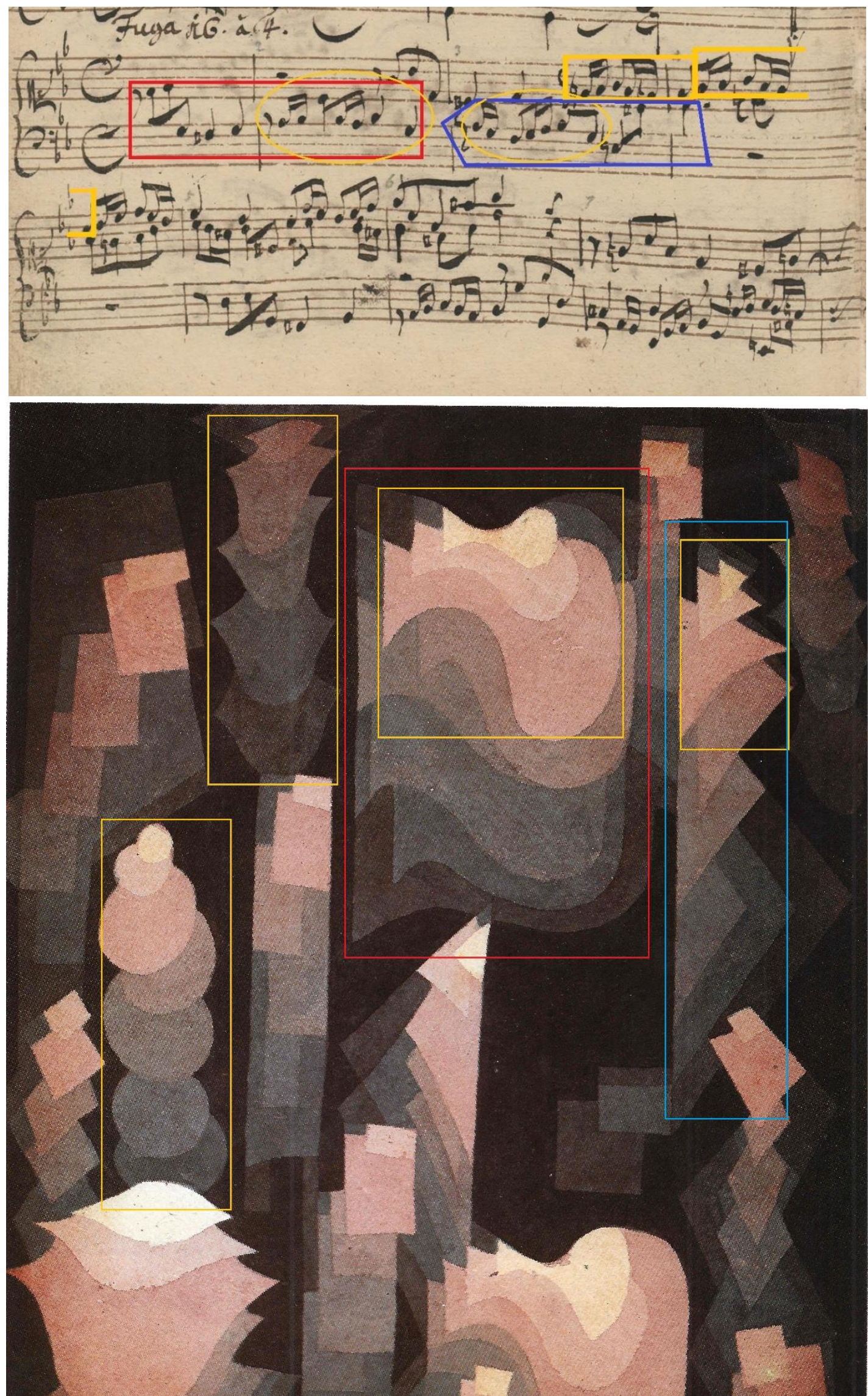

Figures 3a e 3b: L'effet du tableau suggéré par Boult

\section{MUSICA THEORICA}

Revista da Associação Brasileira de Teoria e Análise Musical Journal of the Brazilian Society for Music Theory and Analysis @ TeMA 2018 - ISSN 2525-5541 
Même si l'on considère l'analogie avancée par Sir Adrian Boult ([s.d.], p. 21), qui disait que le public doit avoir l'impression de regarder une grande partition d'un seul coup d'œil, l'interprète doit évidement respecter l'ordre des événements musicaux tels qu'ils se présentent dans la partition. Le même phénomène ne s'applique pas quand on regarde le tableau de Klee (Figures 3a et $3 b)$. L'analogie fonctionne, comme nous l'avons déjà dit, en fonction de la formation musicale du récepteur, mais cela ne voudrait pas dire que nous devons essayer de « lire » le tableau dans une certaine direction, en courant le risque de laisser passer la magie caractéristique de l'impact visuel.

\section{2 - Pastorale}

Dans cette oeuvre, l'analogie est directement liée à la notation musicale; selon Hall (1992, p.68), les symboles, distribués horizontalement à travers les lignes parallèles, peuvent être comparés aussi bien à des notes de musique qu'à des lettres, suggérant plusieurs interprétations.

L'analogie avec l'écriture musicale peut se prouver de façon évidente pour les musiciens, permettant une expérience particulière, différente de celle d'un non musicien, mais il s'agit sans doute d'une œuvre visuelle ouverte à plusieurs interprétations, pas nécessairement restreintes au domaine musical. La propre définition du mot «pastorale » inclut une telle pluralité de liaisons, puisqu'il s'agissait de la forme littéraire préférée de la Renaissance avant de devenir la forme poétique prédominante en Italie à la fin du $16^{\mathrm{e}}$ siècle. La poésie pastorale est même considérée comme « la dernière étape du madrigal et le premier pas en direction à l'opéra » (Grout \& Palisca 1988, p. 357). Cette proposition peut être vérifiée dans l'image de Klee, avec la suggestion du paysage bucolique, la musique qui se révèle comme une forme d'expression presque verbale, donnant de la substance à la vision des poètes. Pastorale (1927) évoque l'enregistrement d'un discours, les différents plans sonores - quatre dans le premier système, trois dans le seconde - suggérant les fragments parallèles encadrés par le groupement en forme de contour continu d'un même élément. Si l'on regarde le tableau de haut en bas, cette impression de discours devient plus évidente (Figure 4, ci-dessous). 


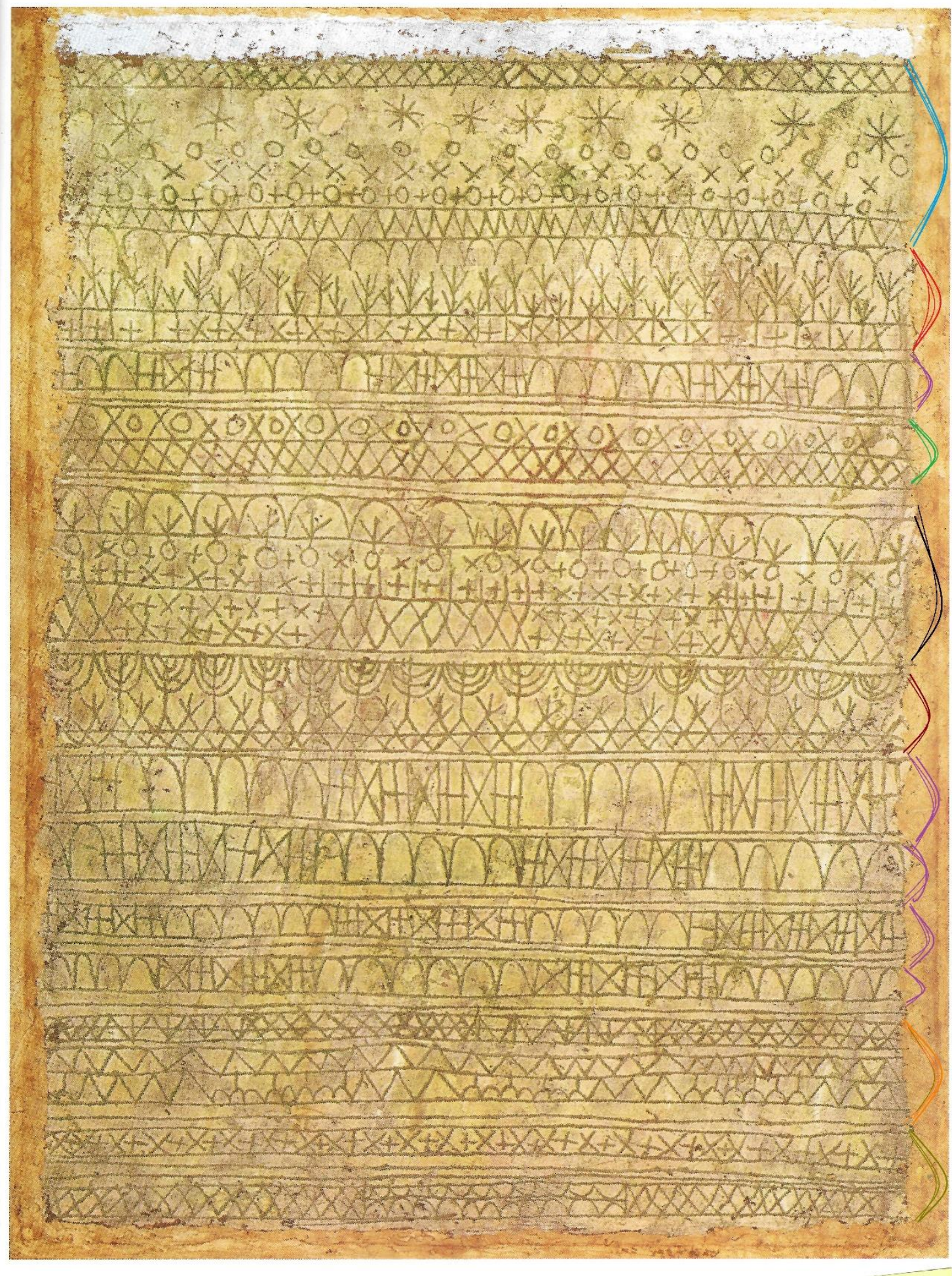

Figure 4: Pastorale de Paul Klee en 12 systèmes

Après les deux premiers systèmes (le $1^{\mathrm{e}}$ indiqué en bleu et le $2^{\mathrm{e}}$ en rouge), on perçoit un troisième système (indiqué en violet) sous la forme d'une ligne 
unique, solide, où l'on regroupe séparément les courbes et deux types différents de croix, chacune dans un carré $(1$ croix +2 courbes +3 croix +5 courbes +7 croix +5 courbes +5 courbes +3 croix $)$.

Un quatrième système (indiqué en vert) combine deux lignes pleines, représentant deux plans sonores parallèles, indépendants, qui ne s'entrelacent pas comme dans les deux premiers systèmes mais gardent un point de liaison grâce aux croix, présentes dans les deux plans. Le cinquième système (indiqué en noir), est plus complexe avec trois lignes pleines indépendantes : la première combine des flèches entourées par des courbes, la deuxième combine deux plans sonores intercalés comme en haut du tableau, entremêlant des cercles avec les deux types de croix. Celles-ci sont séparées par des lignes qui les regroupent $(1+$ $1+9+1+1+1+1+1+1+1+11+1+1+1+1+1+1)$ comme si s'était des « barres de mesure ». La troisième ligne opère un vrai contrepoint, en regroupant deux plans sonores en cinq groupes: dans le premier, les lignes se touchent en formant des diamants; dans le deuxième, les deux types de croix utilisées dans d'autres parties du tableau s'intercalent, le troisième groupe montre à nouveau les diamants; les groupes s'organisent de cette façon jusqu'à la fin du système.

Le sixième système (toujours dans le Figure 4, indiqué en marron) présente un nouveau contrepoint qui utilise les courbes inverties, les croix, les flèches et les lignes, les liant et formant une trame complexe et indéchirable. À partir d'ici, les systèmes se présentent comme dans le troisième (et sont donc indiqués aussi en violet) avec une seule ligne, pleine; l'onzième système (indiqué en orange) ressemble à un stretto, combinant les motifs rencontrés auparavant dans les $1^{\mathrm{er}}, 2^{\mathrm{e}}$ et $3^{\mathrm{e}}$ systèmes. Le douzième système (indiqué en vert foncé) nous rappelle le quatrième dans sa structure, par sa combinaison de deux lignes qui représentent à leur tour deux plans sonores parallèles indépendants gardant une liaison à travers un point commun, la croix; il évoque le troisième système qui agit ainsi comme le point de liaison dans l'ensemble du tableau. La notion d'unité est fortement percevable.

La régularité dans l'organisation des fragments peut être liée à l'association que propose The Oxford Companion to Music entre le terme Pastoral et les mesures de neuf ou douze temps (Scholes 1943, p. 692).

\section{3 - Polyphonie}

Klee combine points et carrés organisés de façon contrapuntique, intercalant divers tons et couleurs, comme dans les plans sonores des ouvres polyphoniques. Nous pouvons distinguer et regrouper les motifs, les points en commun, les intersections, les pauses, puis les contours, unifiant les plans différents et l'œuvre comme un tout. Hall (1992, p.96) souligne la différence entre l'œuvre Polyphonie de 1932 et une antérieure, de 1931 (Ibid., p.92) où l'artiste 
construit une polyphonie à partir d'une seule ligne qui se croise avec elle-même, tout en créant une seule forme (Figure 5a).

Cette approche peut être comparée à la polyphonie linéaire présente dans plusieurs oeuvres des différentes périodes; par exemple, les contours mélodiques dans la musique de Mozart ou dans les suites pour violoncelle de Bach, ce dernier cas de figure est plus évident à travers les transcriptions de Bach lui-même pour luth, révélant les différentes voix en deux pentagrammes (Figure 5b).

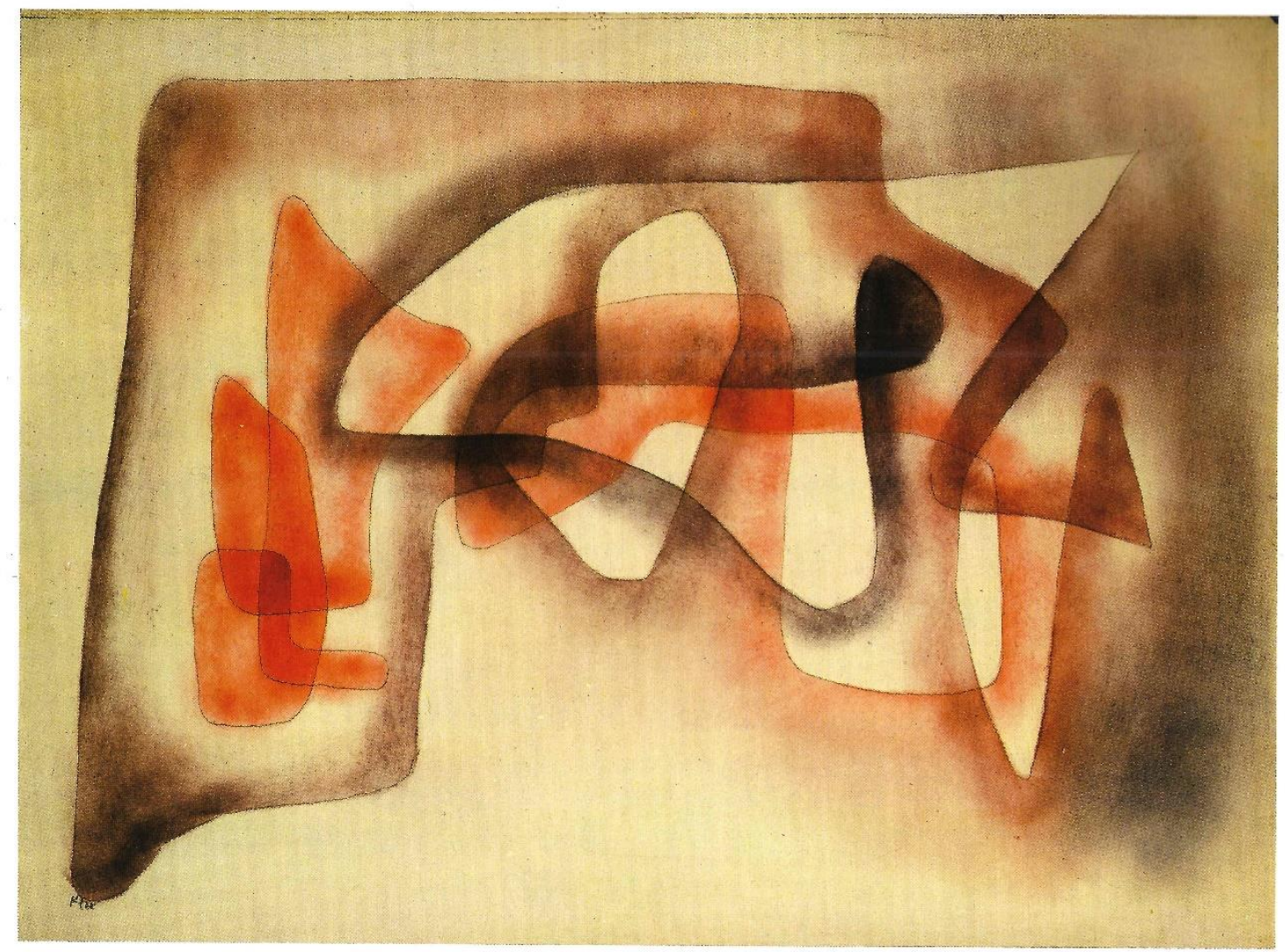

Figure 5a: Paul Klee - Three subjects, Polyphony
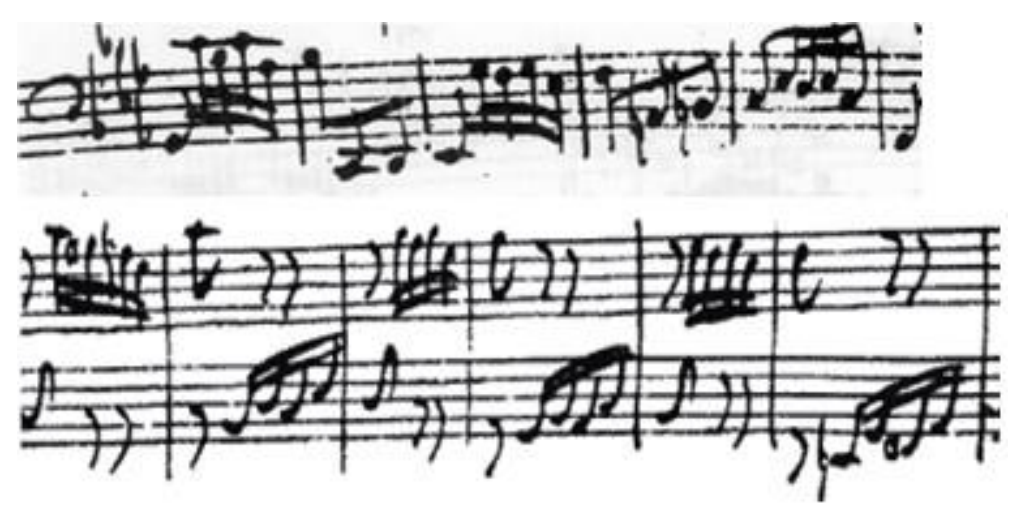

Figure 5b: J. S. Bach, Suite no 5 pour violoncelle solo BWV 1011 - Prélude $\left(^{*}\right)$ 
${ }^{*}$ Note sur la Figure 5b: Autographe de J. S. Bach (copie par Ana Madalena Bach) et transcription pour lute en sol mineur BWV 995.

L'œuvre de 1932 présente le principe de la polyphonie à travers les différents plans de couleurs, qui ne sont définis par aucune ligne. Par contre, comme dans les œuvres polyphoniques, on peut déceler une ligne de cohérence à partir des points en commun liant les différents motifs, par exemple le contour formé par les carrés bleus avec les points bleus qui s'intercalent avec le contour formé par les carrés bleus avec les points beiges présents en haut du tableau, à gauche. Si l'on continue le groupement horizontal, nous découvrons une trame qui nous transporte à travers une forme de narration visuelle, avec ses plans parallèles, son contrepoint et les interruptions éventuelles avec les carrés de couleurs différentes du contexte où ils sont inclus. On peut y trouver une allusion avec plusieurs œuvres musicales, mais les contours s'entrelaçant dans le dernier Intermezzo op.116 de Brahms (Exemple 2), nous semble visuellement très proche de l'idée de Klee.

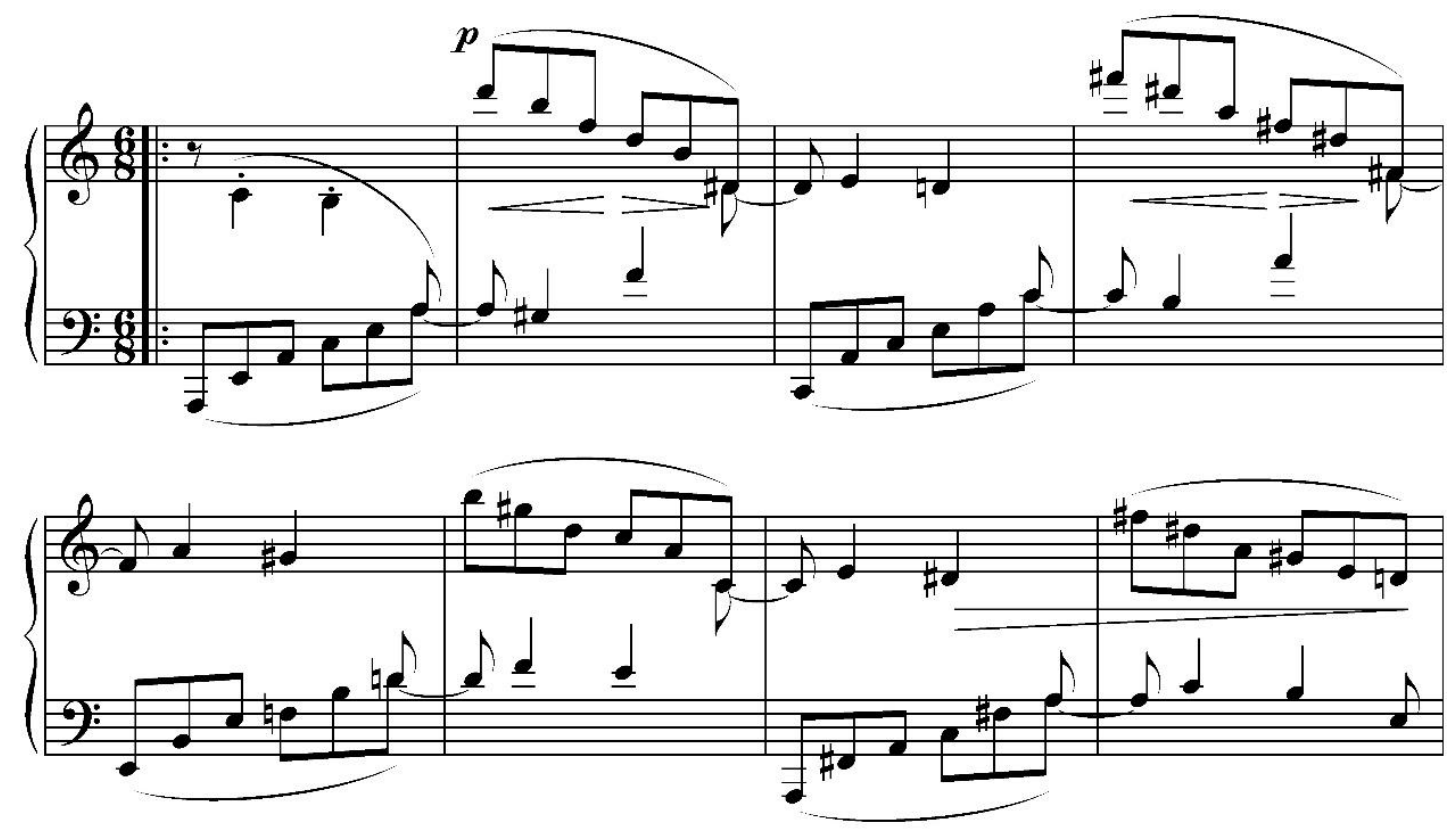

Exemple 2: Brahms, Intermezzo op.116 no 7 mes. 25-28

\section{3 - Synthèse: Liberté}

L'amitié et la collaboration entre les artistes de différentes spécialités sont connues durant toutes les périodes de l'histoire. Pour prendre l'exemple de la Belle Époque, Paris était un centre névralgique: Debussy, Ravel, Toulouse- 
Lautrec, Monet et Nijinsky, entre autres, fréquentaient les mêmes cafés, cet échange devenant évident dans une partie assez significative de leur production.

Tous les arts utilisent des symboles, qui construisent des langages spécifiques de beauté et de qualité évidentes. La fonction de l'artiste et des amateurs d'art est celle de promouvoir l'individualité, en profitant des aspects uniques de chaque œuvre d'art. S'agissant d'un univers subjectif représenté - dans la mesure du possible - par des signes7, ceux-ci rendent possible une approche objective auquel nous avons recours en faisant référence à des aspects spécifiques. Ce que l'on n'explique pas et qui se prouve impossible d'être verbalisé, constitue exactement la magie, l'essence de l'œuvre d'art, l'insaisissable. Cela dit, le dialogue ente les arts se propose de surpasser tout empêchement et de mettre en valeur l'expérience des nos ancêtres les plus anciens, qui ont dessiné, chanté et dansé en communion avec leur environnement. Une véritable célébration de liberté.

\section{Références}

1. Bach, Johann Sebastian. 1685-1750. Forty-Eight Preludes and Fugues, Book I. London: The Associated Board of the Royal Schools, edited by Donald Francis Tovey, p. 126-127.

2. Boulez, Pierre. 2008. L'écoute de Paul Klee. In: Puchala, Véronique (ed.). Pierre Boulez à voix nue, p. 44-52. Paris: Symétrie.

3. Bosseur, Jean-Yves. 2005. Du son au signe. Paris: Editions Alternatives.

4. Boult, Sir Adrian. [s.d.]. A Handbook on the Technique of Conducting, Oxford: Hall the Printer Limited.

5. Carducci-Augustin, Edgar. (1 ${ }^{\mathrm{er}}$ novembre) 1926. L'idée du thème musical. Paris: Le Courrier Musical

6. Dusapin, Pascal. 2012. Flux, Trace, Temps, Inconscient. Collection Psyché. Nantes: Editions Nouvelles Cécile Defaut.

7. Erben, Walter. 1998. Miró. Köln: Benedikt.

\footnotetext{
7 À ce sujet, dans le domaine de la musique, voir les témoignages des compositeurs tels celles de Pascal Dusapin (2012) et de Jean-Yves Bosseur (2005) sur les limitations de la notation musicale.
} 
8. Goubault, Christian. 2002. Claude Debussy la musique à vif. Paris: Minerve.

9. Grout, Donald Jay and Claude Palisca. 1988. A History of Western Music. New York: W.W. Norton.

10. Hall, Douglas. 1992. Klee. London: Phaidon Press Limited.

11. Lessing, Gotthold Ephraim. 1990. Laocoon. Collection Savoir: Sur L'Art, XIII. Paris: Hermann

12. Magalhães, Homero. 1988. Bach: Prelúdios e Fugas I. São Paulo: Editora Novas Metas.

13. Scholes, Percy. 1943. The Oxford Companion to Music. New York: Oxford University Press. 